Relations industrielles

Industrial Relations

\title{
Cazeneuve, Jean (sous la direction de), La sociologie et les \\ Sciences de la Société, Paris, C.E.P.L., 1975, 540 p.
}

\section{Gilles Dussault}

Volume 32, numéro 1, 1977

URI : https://id.erudit.org/iderudit/028774ar

DOI : https://doi.org/10.7202/028774ar

Aller au sommaire du numéro

Éditeur(s)

Département des relations industrielles de l'Université Laval

ISSN

0034-379X (imprimé)

1703-8138 (numérique)

Découvrir la revue

Citer ce compte rendu

Dussault, G. (1977). Compte rendu de [Cazeneuve, Jean (sous la direction de), $L a$ sociologie et les Sciences de la Société, Paris, C.E.P.L., 1975, 540 p.] Relations industrielles / Industrial Relations, 32(1), 146-147.

https://doi.org/10.7202/028774ar

Tous droits réservés @ C Département des relations industrielles de l'Universite Laval, 1977
Ce document est protégé par la loi sur le droit d'auteur. L’utilisation des services d'Érudit (y compris la reproduction) est assujettie à sa politique d'utilisation que vous pouvez consulter en ligne.

https://apropos.erudit.org/fr/usagers/politique-dutilisation/ 
se that induces dissatisfaction, but the linking of arbitration to other procedures such as limiting access to arbitration to union registration in Great Britain in the 1971 Industrial Relations Act». Quant à nous, cette observation n'a pu nous faire oublier les nombreuses remarques faites à plusieurs reprises dans l'ouvrage du genre «increasing dissillusion about the process in Jamaica and Australia», "failure» du système australien, «undesirable but sometimes necessary evil», et autres. Enfin, il y a lieu de noter que les auteurs insistent particulièrement sur le fait que dans les pays où il $\mathrm{y}$ a «free collective bargaining», l'arbitrage obligatoire des différends ne peut être viable que si toutes les parties acceptent de vivre avec un tel système.

Compulsory Arbitration est, en définitive, un ouvrage qui ne peut que soulever un immense intérêt. Nous terminons par cette remarque fort lucide contenue au dernier paragraphe du volume: "Little can be gained at this point from arguing whether compulsory arbitration is an appropriate part of industrial relations. Rather, the questions for each country are: When should compulsory arbitration be adopted, and what are the likely implications of alternatives structures? Some of the answers may be found in the experience recounted here. The remainder will come from experiences that lie ahead».

\section{Rodrigue BLOUIN}

\section{Université Laval}

Personnal Management. A Human Resource System Approach, by Elmer $\mathrm{H}$. Burack et Robert D. Smith, New York, West Publishing C., N.Y., 1977, 513 pp.

Cet ouvrage, comme le titre l'indique, couvre tout le domaine de la gestion des ressources humaines, et cela dans un contexte économique et juridique toujours changeant, ce qui a incité les auteurs à organiser les connaissances en matière de gestion des ressources humaines en prenant comme point de départ les changements majeurs qui affectent l'entreprise et l'utilisation de ses différentes catégories de personnel. Ceci explique la présence de chapitres nouveaux qui traitent de l'insertion des jeunes au marché du travail, de la gestion du changement, de la gestion des carrières, des nouvelles législations touchant les minorités et l'emploi toujours croissant d'une main-d'œuvre féminine.

L'ouvrage comprend dix-sept chapitres regroupés sous six sections. La présenta- tion de chaque thème suit de près l'enchaînement logique des activités de base inhérentes à la gestion des ressources humaines à savoir l'acquisition, la conservation, le développement et le contrôle des ressources humaines.

Cet ouvrage excelle surtout par le traitement approfondi qu'il accorde à chaque thème, traitement supporté par une présentation de modèles et de techniques que je qualifierais de sophistiqués, un peu à cause du peu de connaissances que j'en ai.

Le volume se distingue également des autres par la pédagogie utilisée. Chaque chapitre s'ouvre par une liste d'objectifs d'apprentissage et une présentation des concepts-clefs dont on retrouve la définition dans un glossaire qui apparaît à la fin. Chaque chapitre est également suivi d'une liste de questions et d'une abondante bibliographie. Après en avoir fait une lecture rapide. je dois constater que l'ouvrage cherche à mettre à la disposition des lecteurs une somme de connaissances qu'on retrouve dans des revues spécialisées et pourtant peu accessibles à la majorité des praticiens. Donc, l'ouvrage serait utile à quelqu'un qui possède déjà une formation initiale dans le domaine ou quelques années d'expérience et qui veut ajouter un peu plus de "professionalisme" dans ce secteur.

Laurent BÉLANGER

Université Laval

Cazeneuve, Jean (sous la dir. de), La sociologie et les Sciences de la Société, Paris, C.E.P.L., 1975, 540 p.

La formule du dictionnaire encyclopédique semble connaître un certain succès, à voir le nombre de publications de ce type sur le marché. L'ouvrage, publié sous la direction de $M$. Jean Cazeneuve, est le deuxième portant sur la sociologie (cf. Cazeneuve J. et Victoroff D. (éd.), $L a$ Sociologie, Paris, C.E.P.L., 1970 et Gérard et Cie, 1977). Inutile de préciser que les répétitions sont nombreuses; toutefois les deux ouvrages sont suffisamment différents pour obliger le lecteur intéressé à se les procurer tous les deux - ce qui n'est pas pour déplaire à l'éditeur - - . On retrouve donc près de 300 mots, expressions et noms d'auteur dont la définition et la présentation constituent la partie $«$ dictionnaire $»$ de l'ouvrage et dix articles qui abordent autant d'aspects de la démarche sociologique. La rédaction de ces articles a été confiée à des chercheurs de longue expérience et ont, dans la plupart des cas, une valeur pédago- 
gique certaine. Citons à titre d'exemples les contributions de MM. François Perroux, «Activité économique et science économique» (pp. 14-74), Claude Rivière, «Le changement social», (pp. 316-337) et Émile Poulat, «Sociologie religieuse» (pp. 399414). Signalons aussi deux articles sur les organisations (Erhard Freidberg, pp. 346365 ) et sur la sociologie du travail (Roland Guillon, pp. 509-532) qui peuvent constituer des introductions intéressantes à ces thèmes.

Il ne faut pas s'attendre à trouver dans cet ouvrage «le bilan le plus actuel qui soit des recherches en cours " en sciences sociales qui nous promet la jaquette du livre; on y présente des synthèses honnêtes qui satisferont le profane qui apprendront peu à celui qui est le moindrement familier avec les sujets abordés.

Gilles DUSSAULT

Université laval

Managing Human Resources, par Leonard R. Sayles et George Strauss PrenticeHall Inc., Englewood Cliffs N. J., 1977, $528 \mathrm{pp}$.

Cet ouvrage est un réaménagement et une mise-à-jour d'un volume précédent qui a connu plusieurs éditions et une large diffusion surtout aux États-Unis et au Canada, tellement qu'il n'est pas utile ici de faire une longue présentation. On y retrouve évidemment toute les rubriques qu'on utilise habituellement pour décrire le domaine de la gestion des ressources humaines dans une entreprise syndiquée Quelques chapitres ont été ajoutés pour tenir compte du développement des sciences du comportement et de la législation nouvelle à l'endroit des groupes minoritaires et de l'emploi de la main-d'œuvre féminine. L'ouvrage se distingue de d'autres dans le même domaine par un traitement du sujet en des termes très simples et qui collent de près à la pratique quotidienne des relations industrielles. Les auteurs s'en tiennent à une vision uniquement descriptive de la place d'un service de personnel dans une entreprise, des objectifs poursuivis, et des activités qui y sont accomplies. Pour s'en rendre compte, il suffit de s'arrêter aux nombreux exemples fortement particularisés et insérés dans le texte, ou encore aux incidents qui apparaissent à la fin de la plupart des chapitres.

Ce volume serait surtout utile à ceux qui veulent se donner une formation initiale à la gestion des ressources humaines ou à ceux qui débutent dans cette carrière sans cette formation initiale adéquate. Il serait également souhaitable qu'on en fasse une traduction en français.

Laurent BÉLANGER

Université Laval

Health Economics Symposium: Proceedings of the First Canadian Conference, by R. D. Fraser, Kingston, Industrial Relations Center, Queen's University, 1976, $209 \mathrm{pp}$.

Cet ouvrage rend compte de la première conférence canadienne sur l'économique de la santé, tenue en septembre 1974 à l'Université Queen's. On a voulu, pour cette première, faire un inventaire des questions, reliées à la santé, présentant un intérêt pour l'économiste; cependant c'est le thème de la rémunération des médecins qui revient le plus souvent dans les propos des conférenciers et qui tend à apparaître comme central (R. G. Evans, "Modeling the Economic Objectives of the Physician», pp. 33-46; T. J. Boudreau et J. Y. Rivard, «An Evaluation of Different Methods for Remunerating Physicians», pp. 52-62; P. Ruderman, «The Political Economy of Fee-Setting and the Future of Fee-forService», pp. 75-90 et R. G. Beck, «Some Dynamic Effects of Copayment on Utilization of Medical Services in Saskatchewan », pp. 98-110). Les auteurs s'interrogent sur le paiement à l'acte et ses alternatives - capitation, salariat, vacation, formules mixtes - comme modes de rémunération des professionnels de la santé; ils essaient particulièrement de mesurer leur impact respectif sur les coûts des services ainsi que sur les types de comportements économiques qu'ils engendrent (profils de pratique). De fait, on est loin de l'unanimité quant à la valeur des différentes façons de payer les médecins, mais tous s'entendent pour dire que le choix d'un mode de paiement des services médicaux est plutôt d'ordre politique qu'économique.

L'éditeur, dans sa présentation des textes des conférences, propose au lecteur une introduction générale, fort intéressante, aux dimensions économiques des soins de santé (K. Adams et R. D. Fraser, «An Overview of the Canadian Health Care System», pp. 6-32). Les données qu'h présente illustrent bien l'importance économique qu'a prise la santé depuis une vingtaine d'années au Canada. Par exemple, les dépenses en soins personnels sont passées de 1.5 milliards de dollars à 5.1 milliards de 1960 à 1971, soit une augmentation de 